Bull. Austral. Math. Soc.

VOL. 53 (1996) [299-303]

\title{
ON SQUARES OF JACOBSON RADICAL RINGS
}

\author{
E.R. PUCZYŁOWSKI AND H. ZAND
}

\begin{abstract}
We construct several examples of Jacobson radical rings which are not squares of
\end{abstract} other rings.

Denote by $J$ and $N$ the classes of Jacobson radical rings and nilpotent rings, respectively. Let $J_{2}=\left\{A \mid\right.$ there is a Jacobson radical ring $R$ such that $\left.A=R^{2}\right\}$. Obviously $J$ is equal to the lower radical class determined by $J_{2} \cup N$. This was noted in [5], Theorem 1, in the context of a problem of Divinsky [4] to represent the Jacobson radical as a lower radical class. However it is not clear whether the representation is non trivial (that is, whether $J \neq J_{2} \cup N$ ). In [5] an example showing that $N \nsubseteq J_{2}$ was constructed (which obviously does not clarify the relation between $J$ and $J_{2} \cup N$ ) and the problem of finding more examples in $J \backslash J_{2}$ was raised. In this note we obtain some results which can be used to construct many such examples. They in particular show that $J \neq J_{2} \cup N$.

The question studied in this paper is a special case of the following extension problem: given rings $A$ and $B$, describe all rings $R$ such that $A \simeq I$, where $I$ is an ideal of $R$ and $R / I \simeq B$. For some results and further references concerning that problem and its applications we refer to [1] and [6].

All rings in this paper are associative. To denote that $I$ is an ideal of a ring $R$ we write $I \triangleleft R$. Given a subset $S$ of a ring $A$, we denote by $l_{A}(S)$ and $r_{A}(S)$ the left and right annihilators of $S$ in $A$, respectively.

Proposition 1. Suppose that $P$ is a ring with an identity, $p \in P, l_{P p}(P p)=$ 0 and $P p \triangleleft R$. Then

(i) for every $r \in R$ and $s \in P, s(p r)=(s p) r$;

(ii) $S=\{s \in P \mid s p \in p R\}$ is a subring of $P$;

(iii) $l_{P}(p) \triangleleft S$ and $r_{R}(p) \triangleleft R$;

(iv) there is an isomomorphism $f: R / r_{R}(p) \rightarrow S / l_{P}(p)$ such that

$$
f\left(\left(P p+r_{R}(p)\right) / r_{R}(p)\right)=\left(p P+l_{P}(p)\right) / l_{P}(p) \text {. }
$$

\section{Received 14th June, 1995}

This research was supportet by the Technical University of Białystok, Poland and the Open University, England.

Copyright Clearance Centre, Inc. Serial-fee code: 0004-9729/96 \$A2.00+0.00. 
Proof: (i) Take any $x \in P p$. Since $s, p, p r, x$ and $r x$ are in $P,[s(p r)] x=$ $s[(p r) x]$ and $(s p)(r x)=s[p(r x)]$. On the other hand $p, r, x$ and $s p$ are in $R$, so $p(r x)=(p r) x$ and $[(s p) r] x=(s p)(r x)$. Consequently $[s(p r)-(s p) r] x=0$. Note also that since $P p \triangleleft R, s(p r)$ and $(s p) r$ are in $P p$. These show that $s(p r)-(s p) r \in$ $l_{P p}(P p)=0$, so $s(p r)=(s p) r$.

(ii) Obviously $S$ is an additive subgroup of $P$. Take $s_{1}, s_{2} \in S$. There are $r_{1}, r_{2} \in R$ such that $s_{i} p=p r_{i}, i=1,2$. Now $\left(s_{1} s_{2}\right) p=s_{1}\left(s_{2} p\right)=s_{1}\left(p r_{2}\right)$. By applying (i) we get that $\left(s_{1} s_{2}\right) p=s_{1}\left(p r_{2}\right)=\left(s_{1} p\right) r_{2}=\left(p r_{1}\right) r_{2}=p\left(r_{1} r_{2}\right) \in p R$. Hence $s_{1} s_{2} \in S$.

(iii) Clearly $l_{P}(p)$ is a left ideal of $S$. Now if $s \in S$, then there is $r \in R$ such that $s p=p r$. By applying (i) we get that $\left(l_{P}(p) s\right) p=l_{P}(p)(s p)=l_{P}(p)(p r)=\left(l_{P}(p) p\right) r=$ 0 . Hence $l_{P}(p)$ is also a right ideal of $S$.

Since $p \in P p \triangleleft R$, for every $x \in R$, there is $s \in P$ such that $p x=s p$. By (i), $p\left(x r_{R}(p)\right)=(p x) r_{R}(p)=s\left(p r_{R}(p)\right)=0$. Consequently $R r_{R}(p) \subseteq r_{R}(p)$, so $r_{R}(p)$ is an ideal of $R$.

(iv) Since $p \in P p$ and $P p \triangleleft R$, for every $r \in R$ there exists $s \in S$ such that $s p=p r$. Observe that if $s^{\prime} p=p r$ for some $s^{\prime} \in S$, then $s-s^{\prime} \in l_{P}(p)$. This shows that on putting $g(r)=s+l_{P}(p)$ we get a well defined map $g: R \rightarrow S / l_{P}(p)$. Clearly $g$ is an additive homomorphism of $R$ onto $S / l_{P}(p)$. If $r_{1}, r_{2} \in R$, then by applying (i) we get that $p\left(r_{1} r_{2}\right)=\left(g\left(r_{1}\right) p\right) r_{2}=g\left(r_{1}\right)\left(p r_{2}\right)=\left(g\left(r_{1}\right) g\left(r_{2}\right)\right) p$. Hence $g\left(r_{1} r_{2}\right)=g\left(r_{1}\right) g\left(r_{2}\right)$ and so $g$ is a ring epimorphism of $R$ onto $S / l_{P}(p)$. Clearly $g(P p)=\left(p P+l_{P}(p)\right) / l_{P}(p)$. Moreover ker $g=\{r \in R \mid p r=0\}=r_{R}(p)$. The isomorphism $f: R / r_{R}(p) \rightarrow S / l_{P}(p)$ induced by $g$ is the desired isomorphism.

Corollary 1. Under the notation of Proposition 1 , if $R^{2}=P p$, then $S^{2}=$ $p P+l_{P}(p)$. In particular if $l_{P}(p)=0$, then $S^{2}=p P$.

By applying the above Corollary one can easily find examples of rings in $J \backslash$ $\left(J_{2} \cup N\right)$.

Example 1. Let $A$ be a ring with an identity and $P=A\{x\}$ be the power series ring over $A$ in the indeterminate $x$. For $p=x, P p \in J$ and $l_{P}(p)=0$. We claim that $P p \notin J_{2}$. Indeed, assuming that $P p \triangleleft R$ and $R^{2}=P p$ we would get by Corollary 1 that there is a subring $S$ of $P$ such that $S^{2}=p P$, which is impossible.

EXAMPLE 2. Let $P$ be a commutative local ring with the maximal ideal $M$. Obviously $M \in J$. Since $P / M$ is a field, it follows from Corollary 1 that if $M$ is a principal ideal of $P$ generated by a regular element $p$, then $M \notin J_{2}$ or $M=M^{2}$. However if $M=M^{2}$, then $p=p^{2} x$ for some $x \in P$. Consequently $p(1-p x)=0$ and since $p$ is regular, $1=p x$. Thus $M=P$, a contradiction.

As a particular $\boldsymbol{P}$ one can take any commutative principal ideal domain localised 
at a maximal ideal.

Observe that if $F$ is a field, then $P=F+x^{2} F\{x\}$ is a local ring whose maximal ideal $M=x^{2} F\{x\}$ is not principal. Since $M=(x F\{x\})^{2}, M \in J_{2}$.

Proposition 1 can be also applied to some other cases of the extension problem. For instance we have:

Corollary 2. Suppose that $P$ is a ring with an identity and $p$ is a central regular element of $P$ such that the ring $P / p P$ is reduced. If $p P \triangleleft R$ and $R / p P$ is a nil ring, then $R=p P \oplus I$ for a nil ideal $I$ of $R$.

Proof: By applying Proposition 1 we get that there is a subring $S$ of $P$ such that $p P \subseteq S$ and $S / p P \simeq R /\left(p P+r_{R}(p)\right)$. However $S / p P$ is a reduced ring and $R /\left(p P+r_{R}(p)\right)$, being a homomorphic image of $R / p P$, is a nil ring. Hence both of them are equal to zero. Consequently $R=p P+r_{R}(p)$. Since $p$ is a central element of $P$, by applying Proposition 1 (i), we get that $\left(p P \cap r_{R}(p)\right) p=0$. However $p$ is a regular element of $P$, so $p P \cap r_{R}(p)=0$. Consequently $R=p P \oplus r_{R}(p)$ and, since $R / p P \simeq r_{R}(p), r_{R}(p)$ is a nil ideal of $R$.

Now we shall present another method of finding rings in $J \backslash J_{2}$.

An algebra over a ring $D$ is called a left chain algebra if the left $D$-ideals of the algebra form a chain. In the case when $D$ is the ring of integers left chain algebras are called left chain rings. Obviously every $D$-algebra which is a left chain ring is a left chain $D$-algebra but not conversely. Commutative left chain algebras are called simply chain algebras.

Many examples of left chain rings can be found in $[2,3]$.

Proposition 2. Suppose that $A \in J$ is a left chain algebra over a field $F$. If for a ring $R, A \triangleleft R$ and $R^{2}=A$, then $A^{2}=A$ or $A^{2}=0$.

Proof: Take $0 \neq a \in A$. Suppose first that $a \in F(R a)$. Then for every $b \in A$, $a b \in F(R a) b=R a F b=F(R a b)$ and further $a b \in F(R a b) \subseteq F(R(R a F b))=R^{2} a F b=$ $A a F b=A a b$. Hence, since $A \in J, a b=0$. Consequently $a A=0$.

Suppose now that $a \notin F(R a)$. Then $I=A a+F a \nsubseteq F(R a)$. Since $I$ and $F(R a)$ are left $F$-ideals of $A$ and $A$ is a left chain $F$-algebra, $A a \subseteq F(R a) \subset I$. Since $\operatorname{dim}_{F} I / A a=1, A a=F(R a)$. This implies that $A a \subseteq R a \subseteq F(R a)=A a$, so $R a=A a$. Now $A a=R^{2} a=R(R a)=R(A a) \subseteq A a$. Thus $A a=R A a$ and further $R A a=R^{2} A a=A^{2} a$, which imply that $A a=A^{2} a$. Consequently for every $x \in A$ there exists $y \in A^{2}$ such that $x a=y a$. This gives that $A=A^{2}+l_{A}(a)$. Since $l_{A}(a)$ and $A^{2}$ are left $F$-ideals of $A, l_{A}(a) \subseteq A^{2}$ or $A^{2} \subseteq l_{A}(a)$. In the former case $A=A^{2}$ and we are done. In the latter $A=l_{A}(a)$. This and the conclusion of the last paragraph imply that if $A \neq A^{2}$, then for every $a \in A, A a=0$ or $a A=0$. Thus $l_{A}(A) \cup r_{A}(A)=A$. Consequently $l_{A}(A)=A$ or $r_{A}(A)=A$. The result follows. 
EXAMPLE 3. Let $A=x F[x] / x^{n} F[x]$, where $n$ is an integer $>2$ and $F[x]$ is the polynomial ring over a field $F$ in the interminate $x$. It is easy to check that $A$ is a chain $F$-algebra. Obviously $0 \neq A^{2} \neq A$, so by Proposition $2 A \in J \backslash J_{2}$.

Observe that for $n=2$ the algebra $A$ belongs to $J_{2}$. Indeed, in that case $A \simeq$ $x^{2} F[x] / x^{3} F[x]$ and $x^{2} F[x] / x^{3} F[x]=\left(x F[x] / x^{3} F[x]\right)^{2}$.

EXAMPLE 4. Let $G$ be a linearly ordered Abelian group (written multiplicatively) and let $P$ be the semigroup of positive elements of $G$. Let $R=F[P \cup\{1\}]$ be the semigroup algebra of the semigroup $P \cup\{1\}$ over a field $F$. Observe that $F[P]$ is a maximal ideal of $R$, so for $S=R \backslash F[P], A=S^{-1} R$ is a local $F$-algebra with the maximal ideal $M=S^{-1} F[P]$. Every principal proper ideal of $A$ is of the form $A p$ for some $p \in P$ and if $p, q \in P, p \leqslant q$, then $A q \subseteq A p$. Hence $A$ is a chain ring. All $F$-ideals of $M$ are ideals of $A$, so $M$ is a chain $F$-algebra. Observe that $A^{2}=A$ if and only if $P^{2}=P$. Thus Proposition 2 implies that if $P^{2} \neq P$, then $A \in J \backslash J_{2}$.

The algebra $A$ in Example 4 is a chain ring. However its ideal $M$ is a chain ring if and only if $F$ is a finite prime field. It is a consequence of the following more general observation.

PRoposition 3. If an algebra $R$ over a field $F$ is a Jacobson radical left chain ring, then $F$ is a finite prime field.

Proof: Let $K$ be the subring of $F$ generated by 1 . It suffices to show that $K=F$. Take $0 \neq r \in R$ and $f \in F$. Observe that $K f r+R r$ and $K r+R r$ are left ideals of the ring $R$. Thus $K f r+R r \subseteq K r+R r$ or $K r+R r \subseteq K f r+R r$. In the former case $f r \in k r+R r$ for some $k \in K$. If $f \neq k$, then $r \in R r$, which contradicts the assumption that $R \in J$. Thus $f=k \in K$. In the later case, $r \in k f r+R r$ for some $k \in K$. Hence $(1-k f) r \in R r$. If $k f \neq 1$, then $r \in R r$, a contradiction. Thus $k=f^{-1} \in F$. Consequently $K=F$ and the result follows.

It is natural to ask whether if $A \in J_{2}$ is a left chain ring, then $A=A^{2}$ or $A^{2}=0$. We close by showing that the answer to this question is negative.

EXAMPLE 5. Let $R$ be the ring of integers localised at the set of odd integers. Clearly $R$ is a commutative local ring with the maximal ideal $M=2 R$. Every non-zero ideal of $R$ is of the form $2^{k} R$ for a non-negative integer $k$. Moreover for every $k$, the additive group of $R / 2^{k} R$ is cyclic of order $2^{k}$. Let $A=M^{2}$. Take an ideal $I$ in $A$ and put $\bar{I}=R I$. Obviously $\bar{I}$ and $4 \bar{I}$ are ideals of $R$, so the additive group of $\bar{I} / 4 \bar{I}$ is cyclic of order 4. However $4 \bar{I}=4 R I=M^{2} I \subseteq I \subseteq \bar{I}$, so $I=\bar{I}$ or $I=2 \bar{I}$ or $I=4 \bar{I}$. In all cases $I \triangleleft R$. Hence $A$ is a chain ring. Clearly $A \in J_{2}$ and $A \neq A^{2} \neq 0$.

\section{REFERENCES}

[1] K.I. Beidar, 'On essential extensions, maximal essential extensions and iterated maximal 
essential extensions in radical theory', in Theory of Radicals (Proc. Conf. Szekszard, 1991), Colloq. Math. Soc. J. Bolyai 61 (North Holland, Amsterdam, 1993), pp. 17-26.

[2] C. Bessenrodt-Timmerscheidt, H.H. Brungs and G. Törner, Right chain rings, Part 1, Schriftenreihe des FB Mathematik 74 (Universität Duisburgi, 1985).

[3] C. Bessenrodt-Timmerscheidt, H.H. Brungs and G. Törner, Right chain rings, Schriftenreihe des FB Mathematik 101 (Universität Duisburg, 1990).

[4] N.J. Divinsky, Rings and radicals (Allen and Unwin, 1965).

[5] Y. L. Lee, 'A note on the Jacobson radical', Proc. Amer. Math. Soc. 118 (1993), 337-338.

[6] E. R. Puczyłowski, 'On essential extensions of rings', Bull. Austral. Math. Soc. 35 (1987), 379-386.

Faculty of Mathematics

University of Warsaw

02-907 Warsaw, Banach 2

Poland

e-mail: edmundp@mimuw.edu.pl
IET and Faculty of Mathematics and Computing The Open University Walton Hall, MiltonKeynes MK7 6AA England

e-mail: h.zand@open.ac.uk 